University of Nebraska - Lincoln

DigitalCommons@University of Nebraska - Lincoln

$8-1-2003$

\title{
Model-Independent Quantum Approach for Intense Laser Detachment of a Weakly Bound Electron
}

\author{
M.V. Frolov \\ Voronezh State University, Voronezh, Russia
}

N. L. Manakov

Voronezh State University, manakov@phys.vsu.ru

E.A. Pronin

Voronezh State University, Voronezh, Russia`

Anthony F. Starace

University of Nebraska-Lincoln, astarace1@unl.edu

Follow this and additional works at: https://digitalcommons.unl.edu/physicsstarace

Part of the Physics Commons

Frolov, M.V.; Manakov, N. L.; Pronin, E.A.; and Starace, Anthony F., "Model-Independent Quantum Approach for Intense Laser Detachment of a Weakly Bound Electron" (2003). Anthony F. Starace Publications. 85.

https://digitalcommons.unl.edu/physicsstarace/85

This Article is brought to you for free and open access by the Research Papers in Physics and Astronomy at DigitalCommons@University of Nebraska - Lincoln. It has been accepted for inclusion in Anthony F. Starace Publications by an authorized administrator of DigitalCommons@University of Nebraska - Lincoln. 


\title{
Model-Independent Quantum Approach for Intense Laser Detachment of a Weakly Bound Electron
}

\author{
M.V. Frolov, ${ }^{1,2}$ N. L. Manakov, ${ }^{2}$ E. A. Pronin, ${ }^{2}$ and Anthony F. Starace ${ }^{1}$ \\ ${ }^{1}$ Department of Physics and Astronomy, The University of Nebraska, Lincoln, Nebraska 68588-0111, USA \\ ${ }^{2}$ Department of Physics, Voronezh State University, Voronezh 394006, Russia
}

(Received 2 April 2003; published 1 August 2003)

\begin{abstract}
We present a model-independent theory for laser detachment of a weakly bound electron having a nonzero angular momentum. Our treatment reduces to the well-known Keldysh result for tunnel ionization upon neglecting rescattering effects. Numerical results for the above-threshold detachment spectrum of a negative ion having an outer $p$ electron show significant modification of the rescattering plateau as compared to that for an ion having an outer $s$ electron.
\end{abstract}

PACS numbers: $32.80 . \mathrm{Rm}, 32.80 . \mathrm{Gc}$

Many effects of intense laser interactions with atoms and ions have their origin in the basic processes of abovethreshold ionization (ATI) or detachment (ATD), in which the electron spectrum exhibits a series of peaks, separated by the photon energy, and having nearly equal intensity over a large energy region (the so-called "plateau" region). Key features of ATI spectra have been understood using classical, semiclassical, or approximate quantum analyses and calculations [1-3]. These indicate that the low-energy ATI peaks result from direct ionization by tunneling [and hence their intensity may be estimated using the Keldysh approximation (KA) [4]], while the high-energy ATI peaks on the plateau result from inelastic, laser-assisted electron scattering from the atomic core. An accurate quantum treatment may be carried out for one-electron systems, either numerically (the results of which support the single active electron origin of plateaus in ATI spectra [5]) or by use of simple analytical model potentials. A fruitful model for analysing strong field effects in negative ions beyond the KA is the zero-range potential (ZRP) model, which permits an accurate, ab initio formulation of the problem [6] as well as essentially exact numerical results [7]. However, the ZRP is valid only for $s$-electron initial states and does not allow one to analyze the dependence of ATD features on the initial state symmetry. This symmetry is important already in the KA [8]: in this approach it determines the interference of two saddle-point contributions to the ATD rate that result in a significant dependence of the photoelectron angular distributions (ADs) on the initial state parity.

In this Letter, we present a general approach for the description of strong laser detachment of a weakly bound electron having an initial angular momentum $l$ in a shortrange potential well. Our treatment combines the effective range approach of Refs. $[9,10]$ (in which they treat a weakly bound electron with arbitrary $l$ subjected to a static perturbation) with the quasistationary quasienergy states (QQES) approach of Ref. [6] (in which they treat an $s$ electron bound in a ZRP in the presence of a strong laser field). Our analysis shows that the existence of a highenergy (rescattering) plateau is a general feature for an arbitrary initial $l$. However, the interference pattern at the onset of the plateau, the height of the high-energy plateau relative to the low-energy (KA) part of the electron spectrum, and the shape of the ADs all depend significantly on the initial state symmetry. The results presented here are thus more appropriate than ZRP-based predictions of either ATI features for inert gases (other than He) or ATD features of negative ions having valence $p$ electrons (for which experiments are in progress [11]).

In the QQES approach, the decay of a bound state, $\psi_{0}(\mathbf{r})$, in the potential $U(r)$ having the energy $E_{0}=$ $-\left(\hbar^{2} \kappa^{2}\right) / 2 m$ and subjected to a monochromatic laser field $\mathbf{F}(t)=\mathbf{F} \cos \omega t$ is described by the periodic in time QQES wave function $\Phi_{\epsilon}(\mathbf{r}, t)$, which satisfies outgoing-wave boundary conditions. It is the solution of the eigenvalue Schrödinger equation [12],

$$
\left[i \hbar \frac{\partial}{\partial t}+\epsilon+\frac{\hbar^{2}}{2 m} \Delta-U(r)-|e| z F \cos \omega t\right] \Phi_{\epsilon}(\mathbf{r}, t)=0,
$$

for the complex quasienergy, $\epsilon=\operatorname{Re} \epsilon-i \Gamma / 2$, where $\Gamma$ is the total decay rate of the state $\psi_{0}(\mathbf{r})$.

We assume that the potential $U(r)$ supports a shallow bound state $\psi_{0}(\mathbf{r})$, having angular momentum $l$, and vanishes outside a radius $r \approx r_{c}$. Owing to the known asymptotic form of an initial bound state $\psi_{0}(\mathbf{r})$,

$$
\left.\psi_{0}(\mathbf{r})\right|_{r \rightarrow \infty} \rightarrow C_{l} r^{-1} e^{-\kappa r} Y_{l m}(\hat{r}),
$$

our assumption of a shallow bound state means that $\kappa r_{c} \ll 1 . E_{0}$ and $C_{l}$ are regarded as parameters of the problem. To obtain the complex quasienergy $\epsilon$, we generalize the method used in Refs. [9,10] for timeindependent Hamiltonians to our time-dependent case. The general idea is that at small $r\left(r \lesssim r_{c}\right)$ the interaction potential with the laser field may be neglected compared to the binding potential $U(r)$, whereas at large $r\left(r>r_{c}\right)$ we can construct the general solution of Eq. (1) as a wave 
packet composed of free electron states in a laser field, i.e., for $U(r)=0$. The equation for $\epsilon$ may then be obtained by properly matching the solutions of Eq. (1) in the small- $r$ and large- $r$ regions at some point $r \gtrsim r_{c}$. The key simplification is that the solution of Eq. (1) inside a shortrange potential $U(r)$ (i.e., for $r \leqslant r_{c}$ ) is independent of the shape of $U(r)$, in accord with effective range theory. Since any solution of Eq. (1) has the quasienergy form, $\Phi_{\epsilon}(\mathbf{r}, t)=\sum_{s} \Phi_{\epsilon}^{s \omega}(\mathbf{r}) \exp (-i s \omega t)$, we represent it for small $r$ as follows (cf. [10,13]):

$$
\begin{aligned}
\Phi_{\epsilon}(\mathbf{r}, t) \sim & Y_{l m}(\hat{r}) \sum_{s}\left[r^{-l-1}+\cdots+r^{l} B_{l}(\epsilon+s \hbar \omega)\right] f_{s} \\
& \times \exp (-i s \omega t),
\end{aligned}
$$

where the $f_{s}$ are Fourier-coefficients of a periodic function $f_{\epsilon}(t)=\sum_{s} f_{s} \exp (-i s \omega t)$, and where

$$
\begin{gathered}
(2 l-1) ! !(2 l+1) ! ! B_{l}(E)=-1 / a_{l}+r_{l} k^{2} / 2, \\
k^{2}=2 m E / \hbar^{2} .
\end{gathered}
$$

The parameters $a_{l}$ and $r_{l}$ are the scattering length and the effective range [13], which may be expressed in terms of $\kappa$ and $C_{l}[9,10]$. Thus, the wave function (3) retains the spatial symmetry of the initial state $\psi_{0}(\mathbf{r})$ [cf. Eq. (2)]. However, its radial dependence involves the irregular solution $\left(\sim r^{-l-1}\right.$ at $\left.r \rightarrow 0\right)$ for the potential $U(r)$.

Next, starting from Eq. (1) with $U(r)=0$, we seek a solution which satisfies the outgoing-wave boundary condition for $r \rightarrow \infty$ and has the singularity $\sim r^{-l-1} Y_{l m}(\hat{r})$ at $r \rightarrow 0$. For this purpose we introduce the function

$$
\chi_{\epsilon}\left(\mathbf{r}, \mathbf{r}^{\prime}, t\right)=2 \pi \int d t^{\prime} e^{i \epsilon\left(t-t^{\prime}\right) / \hbar} f_{\epsilon}\left(t^{\prime}\right) G^{(+)}\left(\mathbf{r}, t ; \mathbf{r}^{\prime}, t^{\prime}\right),
$$

where $G^{(+)}$is the retarded Green's function for a free electron in the laser field $\mathbf{F}(t)$. One easily verifies that $\chi_{\epsilon}(\mathbf{r}, 0, t)$ is the solution of Eq. (1) with $U(r)=0$ having an outgoing-wave form at large $r$ and the singularity $\sim r^{-1}$ at $r \rightarrow 0$. The solution $\Phi_{\epsilon}(\mathbf{r}, t)$ with the proper singularity at $r \rightarrow 0$ may be obtained from $\chi_{\epsilon}\left(\mathbf{r}, \mathbf{r}^{\prime}, t\right)$ by acting on it with the differential operator $Y_{l m}\left(\partial / \partial \mathbf{r}^{\prime}\right)$ and then setting $\mathbf{r}^{\prime}=0$ (cf. Refs. $[9,10]$ ). For $p$ states, the desired solution $\Phi_{\epsilon}(\mathbf{r}, t)$ is proportional to

$$
\left.\left(\frac{\partial}{\partial z^{\prime}}\right)^{1-|m|}\left(\frac{\partial}{\partial x^{\prime}}+i m \frac{\partial}{\partial y^{\prime}}\right)^{|m|} \chi_{\epsilon}\left(\mathbf{r}, \mathbf{r}^{\prime}, t\right)\right|_{\mathbf{r}^{\prime}=0} .
$$

Taking into account the Feynman form for $G^{(+)}$in terms of the classical action $S\left(\mathbf{r}, t ; \mathbf{r}^{\prime}, t^{\prime}\right)$, the QQES wave function for a $p$ state may be presented as

$$
\begin{aligned}
\Phi_{\epsilon}(\mathbf{r}, t)= & C_{1} \frac{\sqrt{3 i}}{8 \pi} \int_{0}^{\infty} d \tau \frac{e^{i \epsilon \tau+i S(\mathbf{r}, t ; 0, t-\tau)}}{\tau^{3 / 2}} \\
& \times\left[\frac{r}{\tau} \sqrt{\frac{4 \pi}{3}} Y_{1, m}(\hat{r})+\delta_{m, 0} \varphi(t, \tau)\right] f_{\epsilon}(t-\tau),
\end{aligned}
$$

where

$$
\varphi(t, \tau)=\frac{2 F}{\omega}\left[\sin \omega(\tau-t)-\frac{2}{\omega \tau} \sin \frac{\omega \tau}{2} \sin \omega\left(\frac{\tau}{2}-t\right)\right] .
$$

In Eq. (6) and below we use the following scaled units: the laser amplitude $F$ is measured in units of $F_{0}=$ $\sqrt{2 m\left|E_{0}\right|^{3}} /|e| \hbar$, and energies and $\hbar \omega$ in units of $\left|E_{0}\right|$. Since the interaction with a laser field destroys the spherical symmetry of the problem, only the initial angular momentum projection, $m$, is conserved; thus, the QQES wave function (6) involves (laser field-induced) angular momentum components with any $l \geq|m|$ [14]. Analysis of Eq. (6) shows that, as for the ZRP model [6], the function $f_{\epsilon}(t)$ involves only even Fourier harmonics, $f_{s}=f_{2 k}$, for both $m=0$ and $|m|=1$. Expanding $\Phi_{\epsilon}(\mathbf{r}, t)$ in Eq. (6) in $r$ up to terms $\sim r$, projecting it onto the spherical harmonic $Y_{1 m}(\hat{r})$, and comparing the result with Eq. (3), we obtain an infinite homogeneous system of linear equations for $f_{2 k}$ and $\epsilon$ :

$$
\left[1-(-\epsilon-2 k \omega)^{3 / 2}+\frac{r_{1}}{2}(1+\epsilon+2 k \omega)\right] f_{2 k}=\sum_{k^{\prime}}\left[M_{k, k^{\prime}}(\epsilon)+\delta_{|m|, 0} \tilde{M}_{k, k^{\prime}}(\epsilon)\right] f_{2 k^{\prime}},
$$

where the matrix elements $M_{k, k^{\prime}}$ and $\tilde{M}_{k k^{\prime}}$ involve integrals of Bessel functions $J_{k-k^{\prime}}(x)$ (cf. [6]).

The $n$-photon ATD amplitude, $\mathcal{A}_{n}$, for electron ejection in the direction $\mathbf{n}=\mathbf{r} / r$ is given by the $n$th Fourier coefficient in the asymptotic form of $\Phi_{\epsilon}(\mathbf{r}, t)$ in Eq. (6) at $|\mathbf{r}| \rightarrow \infty$ and may be presented as (cf. Ref. [7] for $l=0$ ):

$$
\begin{aligned}
\mathcal{A}_{n}^{(|m|)}(\mathbf{n})= & i^{n-1} \sqrt{\frac{3}{4 \pi}} C_{1} k_{n} \sum_{k}(-1)^{k} f_{2 k} \sum_{s=-\infty}^{\infty} J_{s}\left(\frac{u_{p}}{2 \omega}\right) J_{n+2 s-2 k}\left(\frac{2 F k_{n} \cos \theta}{\omega^{2}}\right) \\
& \times\left[\frac{\sin \theta}{\sqrt{2}} \delta_{m, \pm 1}+\left(\cos \theta-\frac{\omega(n+2 s-2 k)}{2 k_{n}^{2} \cos \theta}\right) \delta_{m, 0}\right],
\end{aligned}
$$

where $\theta$ is the angle between $\mathbf{n}$ and $\mathbf{F}, k_{n}=\sqrt{\epsilon+n \omega-u_{p}}$, and $u_{p}$ is the scaled ponderomotive shift $U_{p}=e^{2} F^{2} /\left(4 m \omega^{2}\right)$, i.e., $u_{p}=U_{p} /\left|E_{0}\right|=F^{2} /\left(2 \omega^{2}\right)$. The $n$-photon differential detachment rate is given by

$$
\frac{d \Gamma_{n}^{(|m|)}(\mathbf{n})}{d \Omega}=2\left|\sqrt{k_{n}} \mathcal{A}_{n}^{(|m|)}(\mathbf{n})\right|^{2} .
$$

Our quantum approach justifies analytically the Keldysh result [4] for tunnel ionization (in particular, it resolves a longstanding problem regarding which gauge must be used in Keldysh-like theories [15]) and provides clear evidence of 


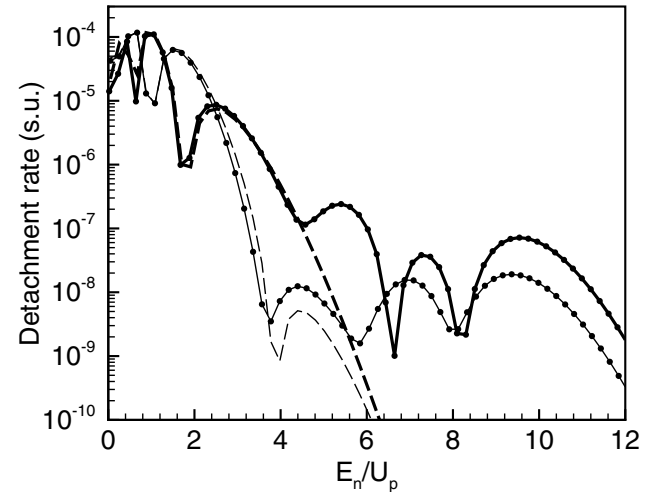

FIG. 1. ATD spectra in scaled units (s.u.) (along the polarization axis, $\theta=0$ ) as a function of electron energy in units of $U_{p}$ for $\mathrm{H}^{-}\left(s\right.$ state; thin lines) and $\mathrm{F}^{-}$( $p$ state; thick lines) at the same scaled parameters: $\omega=0.203, F=0.2835$. Solid lines: exact results; dashed lines: the KA results. Solid circles mark the positions of ATD peaks (up to $n=67$ ). Results for $\mathrm{F}^{-}$ are multiplied by the factor 6.24 in order that the maximum rates are the same in the $\mathrm{H}^{-}$and $\mathrm{F}^{-}$ATD spectra.

"rescattering" effects. Indeed, for not too high $F(F \ll$ 1) and low frequencies $[\gamma \equiv(\omega / F) \ll 1]$, it is reasonable to expect that the time dependence of $f_{\epsilon}(t)$ in Eq. (3) is weak and to retain only the coefficient $f_{s=0}=1$. Then the KA result for $\mathcal{A}_{n}$ follows from our exact Eq. (8) by neglecting all coefficients $f_{2 k}$ except $f_{0}=1$ and setting $\epsilon=E_{0}=-1$. In the KA limit our numerical results (see the dashed curves in Fig. 1) are in close agreement with KA results by Gribakin and Kuchiev [8]. Thus, the KA corresponds to an approximate (particular) solution of Eq. (1), i.e., the wave packet (6) with $f_{\epsilon}=1$ and $\epsilon=$ $E_{0}$. The general solution (6), however, involves higher harmonics of $f_{\epsilon}(t)$ as well, which correspond to the shifted quasienergy, $\epsilon \rightarrow \epsilon+s \omega$ [see Eq. (3)], and, in classical terminology, the terms with $k \neq 0$ in the ATD amplitude (8) describe rescattering effects.

To present our numerical results we choose $\omega=0.203$; this corresponds to $\lambda=1.8 \mu \mathrm{m}$ (or $\hbar \omega=0.689 \mathrm{eV}$ ) for the $\mathrm{F}^{-}$ion (for which $\left|E_{0}\right|=3.4 \mathrm{eV}$ and $C_{1}=0.84$ a.u.), as in the experiment [11]. We present also results for $\mathrm{H}^{-}$ for the same scaled laser parameters $\omega$ and $F$ (note that the scaled unit of intensity $I=F^{2}$ for $\mathrm{F}^{-}$is $I_{\mathrm{F}^{-}}=1.37 \times$ $10^{14} \mathrm{~W} / \mathrm{cm}^{2}=91.5 I_{\mathrm{H}^{-}}$). Figure 1 shows a number of marked differences between results for $p$ and $s$ states in the strong field regime (for the Keldysh parameter $\gamma=$ $\omega / F=0.715$ ). In both cases the KA breaks down (and thus the onset of the plateau occurs) at approximately the same electron energy (i.e., $\sim 3.8 U_{p}$ for the $s$ state and $\sim 4.4 U_{p}$ for the $p$ state) [16] and the plateau cutoffs correspond to the well-known classical estimate, $\sim 10 U_{p}$ [3]. However, the interference pattern near the onset energy is much more pronounced for $\mathrm{F}^{-}$, and the height of the plateau (relative to the KA part of the ATD spectrum) for $p$ states exceeds that for $s$ states by an order of magnitude on average.
As a result of interference between "direct" and "rescattered" electrons near the plateau onset, for $p$ states the ATD spectrum in Fig. 1 decreases (on average) less precipitously with energy than for $s$ states. Such behavior, as well as the relation between the KA and high-energy parts of the spectrum, are in better qualitative agreement with experiments for the inert gases [17] (other than He, for which the $s$-state ATD spectrum is more relevant [18]). Figure 2 presents 3D ADs. One sees that the $p$ state $\left(\mathrm{F}^{-}\right)$ AD differs considerably from that for the $s$ state $\left(\mathrm{H}^{-}\right)$. The two ADs have similar shapes only near and beyond the cutoff [where only a few coefficients $f_{2 k}$ with $k \approx$ $k_{\max }$ contribute to the amplitude (8); see below]. On the plateau one observes also a clear periodicity of the ADs with energy (or $n$ ). However, the $\mathrm{AD}$ for $\mathrm{F}^{-}$is much more localized about $\theta=0^{\circ}$ (along the direction of $\mathbf{F}$ ) and exhibits a more pronounced side-lobe structure near the plateau onset.

These results are consistent with the assumption that the relative enhancement of the $p$-electron plateau is caused mostly by a decrease of the KA part of the spectrum with increasing initial angular momentum. Figure 3 presents numerical evidence supporting this
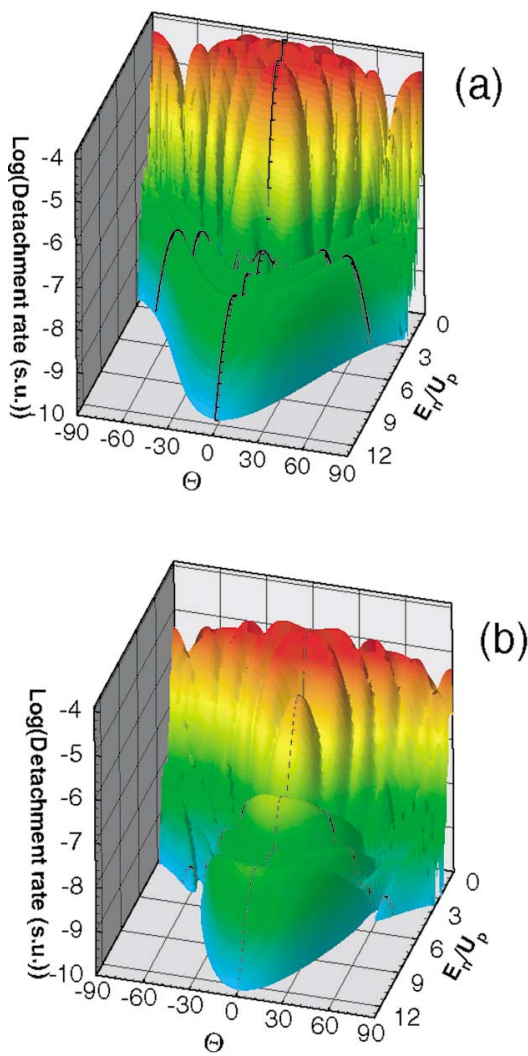

FIG. 2 (color online). Photoelectron ADs for (a) $\mathrm{H}^{-}$and (b) $\mathrm{F}^{-}$for the same laser parameters as in Fig. 1. The solid curves parallel to the $\theta$ axis mark the ADs at the onset of the plateaus, i.e., at $E_{n}=3.8 U_{p}(n=28)$ for the $s$ state and $E_{n}=$ $4.37 U_{p}(n=31)$ for the $p$ state. The first open ATD channel is $n_{0}=10$. 


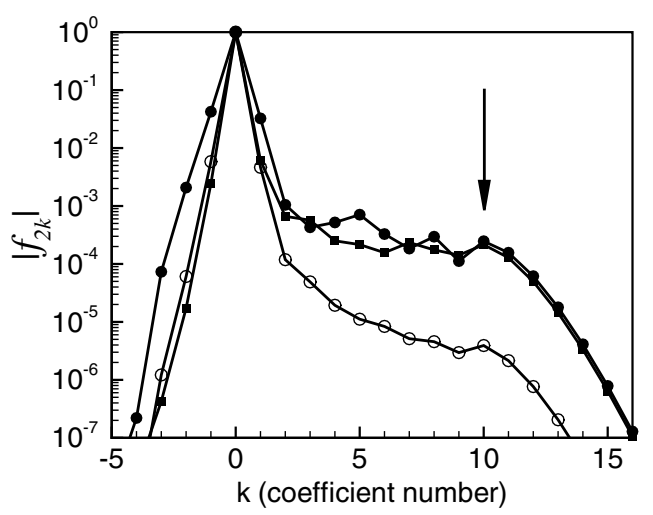

FIG. 3. The spectra of coefficients $f_{2 k}$ for laser parameters as in Fig. 1. Squares: $l=m=0$; solid circles: $l=1, m=0$; open circles: $l=1, m= \pm 1$. The arrow marks the cutoff at $2 k_{\max } \omega \approx 1+3.1732 u_{p}$ (see text).

assumption: the coefficients $f_{2 k}$ for $k \geq 0$ that are responsible for the plateau have about the same magnitude for the cases of $l=0$ and $l=1, m=0$. The suppression of coefficients for $m= \pm 1$ (cf. Fig. 3) is expected, since rescattering stems mostly from the $S$-wave component of (6), whereas for $m= \pm 1$ there is none; rescattering for higher $l$ components is suppressed by the centrifugal barrier. The most spectacular feature of Fig. 3 is the plateaulike structure of the $f_{2 k}$ spectrum, which in turn leads to plateau features in the ATD spectrum. A detailed analysis for different $F$ and $\omega$ predicts the cutoff in the $f_{2 k}$ spectrum to occur at $k \approx k_{\max }$, where (in abs. units) $E_{0}+2 k_{\max } \hbar \omega=\mathcal{E}_{c l}$, and where $\mathcal{E}_{c l}=$ $4 U_{p} \sin ^{2}(\omega \Delta t / 2) \simeq 3.1732 U_{p}$ is the well-known maximum energy of a classical electron, produced with zero velocity in a laser field, that returns to the same point $\mathbf{r}$ after a time interval $\Delta t$ [19]. This connection of the cutoff in the quantum coefficients $f_{2 k}$ with the purely classical quantity, $\mathcal{E}_{c l}$, gives clear evidence of classical features in the behavior of a bound electron in a strong laser field.

In conclusion, we have presented the first quantum analysis of strong field ATD spectra for a weakly bound electron with initial angular momentum $l$ in a shortrange potential $U(r)$. Our treatment encompasses the well-known KA result as a limiting case and demonstrates how the classical rescattering model follows from our quantum analysis. Our predicted enhancement of plateau effects and modification of ADs for valence $p$ electrons may be useful for quantitatively describing negative ion ATD and qualitatively describing rare gas ATI.

This work was supported by NSF Grant No. PHY0070980, by Grant No. E00-3.2-515 of the Russian Ministry of Education, and by the UNL Research Computing Facility.
[1] L. F. DiMauro and P. Agostini, Adv. At. Mol. Opt. Phys. 35, 79 (1995).

[2] M. Protopapas, C. H. Keitel, and P. L. Knight, Rep. Prog. Phys. 60, 389 (1997).

[3] W. Becker, F. Grasbon, R. Kopold, D. B. Milošević, G. G. Paulus, and H. Walther, Adv. At. Mol. Opt. Phys. 48, 35 (2002).

[4] L.V. Keldysh, Zh. Eksp. Teor. Fiz. 47, 1945 (1964) [Sov. Phys. JETP 20, 1307 (1965)].

[5] H. G. Muller and F. C. Kooiman, Phys. Rev. Lett. 81, 1207 (1998).

[6] N. L. Manakov and A. G. Fainshtein, Zh. Eksp. Teor. Fiz. 79, 751 (1980) [Sov. Phys. JETP 52, 382 (1980)].

[7] B. Borca, M.V. Frolov, N. L Manakov, and A. F. Starace, Phys. Rev. Lett. 88, 193001 (2002); N. L. Manakov, M.V. Frolov, B. Borca, and A. F. Starace, J. Phys. B 36, R49 (2003).

[8] G. F. Gribakin and M. Yu. Kuchiev, Phys. Rev. A 55, 3760 (1997).

[9] Yu. N. Demkov and G. F. Drukarev, Zh. Eksp. Teor. Fiz. 81, 1218 (1981) [Sov. Phys. JETP 54, 650 (1981)].

[10] S. P. Andreev, B. M. Karnakov, V. D. Mur, and V. A. Polunin, Zh. Eksp. Teor. Fiz. 86, 866 (1984) [Sov. Phys. JETP 59, 506 (1984)].

[11] I. Yu. Kiyan and H. Helm, in Abstracts of 9th International Conference on Multiphoton Processes (Elounda, Greece, 2002). [Experimental results for the Keldysh part of the $F^{-}$ATD spectrum have now appeared in Phys. Rev. Lett. 90, 183001 (2003).]

[12] N. L. Manakov, V. D. Ovsiannikov, and L. P. Rapoport, Phys. Rep. 141, 319 (1986).

[13] L. D. Landau and E. M. Lifshitz, Quantum Mechanics (Pergamon, Oxford, 1978), 2nd ed.

[14] However, our numerical analysis up to $F \lesssim 0.5$ shows that only photoelectrons with $0 \leq l \leq n$ contribute to the $n$-photon ATD peaks for both odd and even $n$.

[15] The original formulation [4] of the KA is gauge dependent; it used the length gauge based upon physical arguments (see also Ref. [8]). Since our general analysis is gauge invariant, we could have used the velocity gauge form of Eq. (1), in which case the function $\varphi(t, \tau)$ in Eq. (6) does not involve the term $(2 F / \omega) \sin \omega(\tau-t)$. However, in this case it is impossible to match $\Phi_{\epsilon}(\mathbf{r}, t)$ to the small- $r$ form (3). We must thus add "by hand" the missing term to the function $\varphi(t, \tau)$ in the velocity gauge, which is equivalent to starting from the length form.

[16] Note that in our case (for $\hbar \omega /\left|E_{0}\right| \approx 0.2$ ) the plateau onset energy is much higher than the classical estimate, $\sim 2 U_{p}$, which is valid for $\hbar \omega \ll\left|E_{0}\right|[2,3]$.

[17] M. J. Nandor, M. A. Walker, and L. D. Van Woerkom, J. Phys. B 31, 4617 (1998).

[18] B. Walker, B. Sheehy, K. C. Kulander, and L. F. DiMauro, Phys. Rev. Lett. 77, 5031 (1996).

[19] W. Becker, S. Long, and J. K. McIver, Phys. Rev. A 50, 1540 (1994). 